\section{Uso de serviços ambulatoriais por idosos nas regiões Sul e Nordeste do Brasil}

\author{
Use of outpatient services by the elderly in the \\ South and Northeast of Brazil
}

\author{
${ }^{1}$ Faculdade de Medicina, \\ Universidade Federal de \\ Pelotas, Pelotas, Brasil. \\ 2 Secretaria Estadual de \\ Saúde do Rio Grande do Sul. \\ Pelotas, Brasil. \\ 3 Universidade Católica de \\ Pelotas, Pelotas, Brasil. \\ 4 Secretaria Municipal \\ de Saúde e Bem-Estar de \\ Pelotas, Pelotas, Brasil. \\ 5 Faculdade de Enfermagem \\ e Obstetrícia, Universidade \\ Federal de Pelotas, Pelotas, \\ Brasil. \\ Correspondência \\ M. A. P. Rodrigues \\ Programa de Pós-graduação \\ em Epidemiologia, Faculdade \\ de Medicina, Universidade \\ Federal de Pelotas. \\ Rua Marechal Deodoro 1160 \\ $3^{\circ}$ piso, Pelotas, $R S$ \\ 96020-220, Brasil. \\ rodriguesmapa@terra.com.br
}

\section{Abstract}

This study evaluated the use of outpatient services by senior citizens $(N=4,003)$ drawing on data from the baseline study that evaluated the Project for Expansion and Consolidation of the Family Health Strategy (PROESF) in 41 cities in South and Northeast Brazil. Use of outpatient services was greater and more appropriate to the needs of the elderly in the South than in the Northeast. Primary care facilities in both regions met the demand by lower-income elderly, but those requiring more care were treated at other levels. The results highlight the need to increase the supply of outpatient services and ensure access by the elderly, particularly for individuals with functional impairments in the Northeast. In addition to promoting equity, primary care in both regions should adopt targeted approaches for the health needs of senior citizens.

Ambulatory Health Services; Program Evaluation; Primary Health Care; Health of the Elderly
Maria Aparecida Pinheiro Rodrigues 1,2

Luiz Augusto Facchini 1

Roberto Xavier Piccini 1

Elaine Tomasi 3,4

Elaine Thumé 5

Denise Silva Silveira 1,4

Vera Maria Vieira Paniz 1

Fernando Vinholes Siqueira 1,3

\section{Introdução}

O envelhecimento da população é um fenômeno mundial, no entanto nos países não industrializados teve início mais tardio e está ocorrendo em um ritmo mais acelerado. Nesses países está previsto um crescimento de $200 \%$ a $400 \%$ da população idosa nos próximos trinta anos. Os sistemas públicos de saúde desses países terão o desafio de garantir o atendimento às necessidades de saúde dessa população ${ }^{1}$.

A atenção básica à saúde é o primeiro nível de serviços de um sistema de saúde e, portanto, deve ser o principal veículo de oferta de cuidados de saúde aos idosos. Em 2004, a Organização Mundial da Saúde (OMS) elaborou um documento no qual preconiza que na atenção básixa à saúde deve ser oferecido um atendimento adequado às necessidades dos idosos, além de permitir a participação deles nas decisões a respeito da própria saúde. O objetivo foi sensibilizar e educar os trabalhadores do nível primário de saúde sobre as necessidades específicas dos usuários idosos 2 .

No Brasil, todos os indivíduos têm direito ao Sistema Único de Saúde (SUS), que oferece serviços de saúde nos três níveis de atenção 3 . No nível primário há oferta de atendimento em unidades básicas de saúde, que costumam se localizar em áreas periféricas das cidades, para facilitar o acesso dos indivíduos mais pobres, e em serviços ligados a instituições universitárias e hospitais 4 . No sistema privado de saúde, 
há diversas operadoras que oferecem variados planos de saúde mediante pagamento dos associados 5 .

A legislação brasileira de proteção à saúde do idoso teve início em dezembro de 1999 quando foi aprovada a Política Nacional de Saúde do Idoso pelo Ministério da Saúde 6 . Mais tarde, em outubro de 2003, foi criado o Estatuto do Idoso por meio da Lei $n^{o} .10 .741$ 7, que assegurou atenção integral à saúde do idoso garantindo atenção especial às doenças que afetam preferencialmente este grupo populacional, incluindo atendimento geriátrico na atenção básica à saúde. O Pacto pela Vida, incluído no Pacto pela Saúde 2006 8, compromisso firmado pelos gestores do SUS nos âmbitos federal, estadual e municipal, definiu pela primeira vez entre as suas prioridades a implantação da Política Nacional de Saúde da Pessoa Idosa. Apesar desse avanço, a falta do estabelecimento de metas para os gestores inviabilizou a exigência de cumprimento das diretrizes estabelecidas naquele documento.

A literatura sobre o uso de serviços de saúde por idosos no Brasil ainda é escassa. A investigação sobre a utilização de serviços de saúde em dois recentes inquéritos populacionais no Brasil demonstrou que os idosos mais pobres têm piores condições de saúde e, apesar disto, usam menos os serviços de saúde 9 . Um estudo de base populacional, realizado na Região Nordeste, observou que os idosos residentes em áreas periféricas procuraram menos os serviços de saúde, porém utilizaram maior número de consultas médicas do que aqueles residentes em áreas centrais da cidade. Um outro estudo que comparou três regiões administrativas de uma mesma cidade revelou que o efeito da renda familiar sobre o consumo de serviços de saúde por idosos diferiu entre as regiões: na mais rica a renda não modificou o uso de serviços de saúde, enquanto que nas regiões mais pobres o aumento da renda elevou o consumo de serviços de saúde 10. Quanto ao local de consulta, um estudo que avaliou a escolha por idosos, entre a rede pública ou privada, mostrou que a preferência pela rede privada associou-se ao gênero feminino, aumento da idade e da escolaridade, aumento da renda individual e familiar e diminuição do número de membros da família ${ }^{11}$.

A intenção deste estudo é avaliar o uso de serviços ambulatoriais, unidades básicas de saúde e outros locais de atendimento, por idosos residentes em áreas de abrangência de unidades básicas de saúde em municípios com mais de 100 mil habitantes nas regiões Sul e Nordeste do Brasil.

\section{Métodos}

Este trabalho utilizou o banco de dados de idosos, definidos como indivíduos a partir de 65 anos de idade, do Estudo de Linha de Base (ELB) do Projeto de Expansão e Consolidação do Saúde da Família (PROESF) executado em 41 municípios com mais de 100 mil habitantes. Desses, 21 estavam localizados na Região Sul (17 municípios do Rio Grande do Sul e 4 de Santa Catarina) e 20 na Região Nordeste (2 municípios de Alagoas, 3 da Paraíba, 10 de Pernambuco, 2 do Piauí e 3 municípios do Rio Grande do Norte). A seleção da amostra foi realizada em múltiplos estágios. Inicialmente foi calculado o tamanho de amostra necessário para examinar diferenças na efetividade das ações programáticas, segundo o modelo atenção, Programa Saúde da Família (PSF) e tradicional, nas áreas de abrangência das unidades básicas de saúde, totalizando 2.100 indivíduos em cada região geográfica, 4.200 em seu conjunto. Para ampliar a representatividade da amostra populacional e de unidades básicas de saúde (e diminuir o efeito de conglomerado) optou-se por localizar a amostra populacional em cerca de $10 \%$ a $20 \%$ da rede básica instalada em cada município, alcançando o número de 120 unidades básicas de saúde em cada região, num total de 240. A seguir, selecionaram-se aleatoriamente as unidades básicas de saúde, com base em listas elaboradas pela Secretaria Municipal de Saúde de cada cidade, estratificadas por modelo de atenção, proporcionalmente ao tamanho da rede básica municipal. Foram selecionadas duas unidades básicas de saúde com modelo de PSF para uma unidade básica de saúde com modelo tradicional. Ao dividir a amostra de idosos pelo total de unidades básicas de saúde obteve-se uma "quota" de 18 indivíduos a serem localizados na área de abrangência de cada unidade. A localização da amostra foi realizada por meio de visitas consecutivas aos domicílios. Devido à proporção de idosos na população ( $8 \%$ a 10\%) identificou-se a probabilidade de localizar um idoso a cada três domicílios, garantindo uma boa distribuição da amostra na área de abrangência de cada unidade básica de saúde 12 .

Foram identificados e entrevistados 4.003 indivíduos, 1.891 na Região Sul e 2.112 na Região Nordeste. Considerando a Região Sul como referência porque apresentou a menor amostra, a margem de erro para a estimativa de prevalência de consulta médica nos últimos trinta dias foi de 2,1\%. Para avaliação de associações, o estudo teve poder de $80 \%$ para detectar como significativas razões de prevalência de 1,3 ou maiores, para exposições que afetam de $10 \%$ a $90 \%$ da população com um nível de 95\% de confiança. 
As entrevistas domiciliares foram realizadas por 15 supervisores, previamente treinados, utilizando um questionário estruturado e pré-codificado. Estava previsto que caso o idoso não conseguisse responder ao questionário um outro indivíduo residente no domicílio o ajudaria, se a incapacidade fosse parcial, ou responderia a todas as questões a respeito do idoso selecionado, se a incapacidade fosse total.

A utilização de serviços ambulatoriais foi avaliada pelo uso ou não de consulta médica nos trinta dias anteriores à data da entrevista e, no caso de ter consultado, o local da última consulta médica neste período. As alternativas para o local da consulta médica mais recente foram: "não consultou nos últimos trinta dias, posto de saúde de abrangência, outro posto de saúde, Pronto Socorro, ambulatório de hospital/faculdade/sindicato ou empresa, consultório médico por convênio ou plano de saúde, consultório médico particular, Centro de Atenção Psicossocial (CAPS) e outro".

As variáveis independentes deste estudo foram: (a) sócio-demográficas (sexo, cor da pele, situação conjugal, idade, escolaridade e renda familiar - dividida em quintis); (b) tabagismo (nunca fumou, ex-fumante e fumante atual); (c) autopercepção de saúde (regular/ruim e excelente/muito boa/boa); (d) limitação (nenhuma e pouca/média/muita dificuldade/necessidade de ajuda de outra pessoa) para realizar três ou mais de 12 atividades da vida diária (tomar banho, alimentar-se, vestir-se, usar o banheiro, caminhar, passar da cama para uma cadeira, lavar o rosto, empurrar um objeto grande, carregar $5 \mathrm{~kg}$, agachar-se, levantar os braços acima da cabeça e lidar com objetos pequenos); (e) modelo de atenção da unidade básica de saúde (tradicional ou PSF).

Os dados foram digitados utilizando-se o programa Epi Info 6.04 (Centers for Disease Control and Prevention, Atlanta, Estados Unidos) e a análise dos dados foi realizada no programa Stata 9.0 (Stata Corp., College Station, Estados Unidos). O controle de qualidade foi realizado por telefone mediante aplicação de um questionário reduzido, alcançando cerca de $6 \%$ da amostra do estudo. A análise de concordância foi obtida utilizando-se o índice kappa.

As análises foram estratificadas por região geográfica para avaliar a possibilidade de modificação de efeito devido às diferenças contextuais. A análise descritiva mostrou as características da amostra. A análise bivariada utilizou o teste de qui-quadrado de Pearson para avaliar o desfecho com as variáveis independentes. A análise multivariada avaliou o uso de serviços ambulatoriais (unidades básicas de saúde de abrangência ou outros locais) pelos idosos por meio de regressão de Poisson com variância robusta, levando em conta o efeito de conglomerado. Foram utilizados os testes de Wald de heterogeneidade de proporções e de tendência linear para variáveis ordinais, com descrição das razões de prevalências e respectivos intervalos de $95 \%$ de confiança (IC95\%).

A análise ajustada seguiu um modelo conceitual. O modelo proposto compreende quatro níveis de determinação. No primeiro foram consideradas as variáveis sócio-demográficas. No segundo nível, um hábito em relação à saúde, o tabagismo. No terceiro, indicadores da condição de saúde, autopercepção de saúde e limitação para três ou mais atividades da vida diária. No quarto nível, o modelo de atenção básica utilizado pela unidade básica de saúde, PSF ou tradicional. Para controlar os fatores de confusão, o efeito de cada preditor foi controlado para outras variáveis do mesmo nível ou superior com valor de $\mathrm{p} \leq 0,2$. O modelo foi utilizado para analisar os dois desfechos de forma dicotômica: o uso de serviços ambulatoriais e o local de consulta (unidade básica de saúde de abrangência/outro local).

Esta pesquisa foi aprovada pela Comissão de Ética e Pesquisa da Universidade Federal de Pelotas. Os entrevistados foram esclarecidos quanto ao sigilo dos dados pessoais, a confidencialidade das informações e foi garantido a eles o direito de participar ou não da entrevista.

\section{Resultados}

Foram entrevistados 4.003 idosos, um percentual de $95,3 \%$ do total previsto para amostra. Entre os 3.994 idosos que responderam à questão da variável dependente, 486 (12,2\%) foram auxiliados por outra pessoa e $105(2,6 \%)$ necessitaram que outra pessoa respondesse ao questionário.

A Tabela 1 descreve as características da amostra. Nas duas regiões geográficas estudadas o sexo feminino predominou em uma proporção de 3:2 em relação ao masculino. A maioria dos indivíduos da amostra tinha cor de pele branca sendo esta proporção maior no Sul (83\%) do que no Nordeste (58\%). Menos da metade dos indivíduos era casado(a) ou tinha companheiro(a) nas duas regiões geográficas. A distribuição dos idosos por idade foi semelhante nas duas regiões concentrando-se mais nas faixas etárias iniciais. A maioria dos idosos não havia freqüentado escola no Nordeste (62\%), enquanto no Sul este grupo representou menos da metade $(41 \%)$ dos indivíduos. Os primeiros dois quintis de renda familiar constituíram $37 \%$ dos indivíduos no Sul e 46\% no Nordeste. A renda 
Tabela 1

Características da amostra de idosos ( $N=4.003$ ) residentes em áreas de abrangência de unidades básicas de saúde em municípios com mais de 100 mil habitantes das regiões Sul e Nordeste do Brasil. Projeto de Expansão e Consolidação do Saúde da Família (PROESF), 2005.

\begin{tabular}{|c|c|c|c|c|}
\hline \multirow[t]{2}{*}{ Variáveis } & \multicolumn{2}{|c|}{ Região Sul ( $n=1.891$ ) } & \multicolumn{2}{|c|}{ Região Nordeste $(n=2.112)$} \\
\hline & $\mathbf{n}$ & $\%$ & $\mathrm{n}$ & $\%$ \\
\hline \multicolumn{5}{|l|}{ Sexo } \\
\hline Masculino & 713 & 37,7 & 841 & 39,8 \\
\hline Feminino & 1.178 & 62,3 & 1.271 & 60,2 \\
\hline \multicolumn{5}{|l|}{ Cor da pele } \\
\hline Branca & 1.560 & 82,8 & 1.229 & 58,4 \\
\hline Parda & 168 & 8,9 & 546 & 25,9 \\
\hline Preta & 156 & 8,3 & 331 & 15,7 \\
\hline \multicolumn{5}{|l|}{ Situação conjugal } \\
\hline Casado(a)/Com companheiro(a) & 797 & 42,5 & 898 & 42,8 \\
\hline Viúvo(a) & 866 & 46,2 & 920 & 43,9 \\
\hline Separado(a)/Divorciado(a)/Solteiro(a) & 211 & 11,3 & 279 & 13,3 \\
\hline \multicolumn{5}{|l|}{ Idade (anos) } \\
\hline $65-69$ & 636 & 33,6 & 665 & 31,5 \\
\hline $70-74$ & 533 & 28,2 & 527 & 25,0 \\
\hline 75-79 & 369 & 19,5 & 450 & 21,2 \\
\hline 80 ou mais & 353 & 18,7 & 470 & 22,3 \\
\hline \multicolumn{5}{|l|}{ Escolaridade (anos) } \\
\hline 9 ou mais & 75 & 4,0 & 82 & 4,0 \\
\hline $5-8$ & 346 & 18,5 & 220 & 10,5 \\
\hline $1-4$ & 689 & 36,9 & 482 & 23,3 \\
\hline 0 & 757 & 40,6 & 1.288 & 62,2 \\
\hline \multicolumn{5}{|l|}{ Renda familiar (quintil) } \\
\hline $5^{\circ}$ & 383 & 20,2 & 303 & 14,3 \\
\hline $4^{\circ}$ & 372 & 20,0 & 339 & 16,1 \\
\hline $3^{\circ}$ & 437 & 23,0 & 506 & 24,0 \\
\hline $2^{\circ}$ & 174 & 9,0 & 743 & 35,2 \\
\hline $1^{\circ}$ & 525 & 27,8 & 221 & 10,4 \\
\hline \multicolumn{5}{|l|}{ Tabagismo } \\
\hline Nunca fumou & 917 & 48,5 & 818 & 38,8 \\
\hline Ex-fumante & 693 & 36,7 & 965 & 45,7 \\
\hline Fumante & 280 & 14,8 & 327 & 15,5 \\
\hline \multicolumn{5}{|l|}{ Autopercepção de saúde } \\
\hline Regular/Ruim & 1.142 & 60,7 & 1.406 & 67,9 \\
\hline Excelente/Muito boa/Boa & 738 & 39,3 & 665 & 32,1 \\
\hline \multicolumn{5}{|c|}{ Limitação para três ou mais atividades de vida diária * } \\
\hline Sim & 464 & 25,4 & 599 & 29,2 \\
\hline Não & 1.362 & 74,6 & 1.454 & 70,8 \\
\hline \multicolumn{5}{|c|}{ Modelo de atenção da unidade básica de saúde } \\
\hline Tradicional & 784 & 41,5 & 596 & 28,2 \\
\hline Programa Saúde da Família & 1.107 & 58,5 & 1.516 & 71,8 \\
\hline
\end{tabular}

* Foi considerado limitação para atividades da vida diária quando o individuo tinha dificuldade ou necessidade de ajuda de outra pessoa para realizar 3 ou mais dentre 12 atividades - tomar banho, alimentar-se, vestir-se, usar o banheiro, caminhar, transferir-se da cama para uma cadeira, lavar o rosto, empurrar um objeto grande (uma poltrona, por exemplo), carregar $5 \mathrm{~kg}$, agachar-se, levantar os braços acima da cabeça e lidar com objetos pequenos (uma chave, por exemplo).

Nota: o número máximo de valores ignorados foi de 124 na variável atividade da vida diária, 65 na Região Sul e 59 na Região Nordeste. 
familiar de $90 \%$ dos idosos foi de até 1.460 Reais no Sul e de até 1.200 Reais no Nordeste. A proporção de idosos que nunca fumaram foi maior no Sul (49\%) do que no Nordeste (39\%). Em ambas as regiões o percentual de indivíduos com autopercepção de saúde como regular/ruim foi acima de $60 \%$ e o de indivíduos com limitações para três ou mais atividades da vida diária foi inferior a $30 \%$. A quantidade de idosos vinculados a unidades básicas de saúde com modelo de atenção do PSF foi maior no Nordeste (72\%) do que no Sul (59\%).

A proporção de idosos que usaram serviços ambulatoriais nos trinta dias anteriores à entrevista foi de 32,4\% (IC95\%: 30,8-34,0) com um efeito de delineamento de 1,23 . Observa-se que a quantidade de idosos que consultaram foi maior no Sul (36\%) do que no Nordeste (29\%). Nas duas regiões aproximadamente $50 \%$ dos idosos que consultaram utilizaram a unidade básica de saúde de abrangência (Tabela 2).

Na Região Sul, a análise bruta (Tabela 3) demonstrou que os idosos que utilizaram mais os serviços ambulatoriais (unidade básica de saúde de abrangência e outros locais) foram do sexo feminino, com limitação para três ou mais atividades de vida diária, autopercepção de saúde regular/ruim e não-fumantes. Em relação à renda familiar houve diferença entre os locais de consulta: o quintil mais associado ao uso da unidade básica de saúde de abrangência foi o de menor renda enquanto que em outros locais foi o de maior renda. Não houve diferenças no uso quanto à cor da pele, situação conjugal, idade, escolaridade dos idosos e modelo de atenção da unidade básica de saúde.

Na Região Nordeste, a análise bruta (Tabela 3) revelou que os idosos não-tabagistas consultaram mais, tanto na unidade básica de saúde de abrangência quanto em outros locais. Outras características dos idosos variaram quanto ao local de consulta ambulatorial. Os que mais consultaram na unidade básica de saúde de abrangência eram de cor parda, tinham menor escolaridade, não tinham limitação para três ou mais atividades da vida diária, pertenciam aos quintis de menor renda familiar e residiam em área de abrangência de unidades básicas de saúde com PSF. Os idosos que mais consultaram em outros locais eram de cor branca, com nove ou mais anos de escolaridade, estavam no quintil superior de renda familiar, tinham limitação para três ou mais atividades da vida diária, autopercepção de saúde regular/ruim e residiam em área de abrangência de unidade básica de saúde com modelo tradicional. Não houve diferenças no uso quanto ao sexo, situação conjugal e idade dos idosos.

O uso de serviços ambulatoriais, após análise ajustada do modelo (não apresentada), associou-se a ser ex-fumante, ter autopercepção de saúde como regular/ruim e limitação para três ou mais atividades da vida diária na Região Sul, enquanto que na Região Nordeste associou-se a ser ex-fumante e ter autopercepção de saúde como regular/ruim.

O uso da unidade básica de saúde de abrangência, após ajuste para as variáveis previstas no modelo, associou-se à redução de renda familiar e melhor autopercepção de saúde na Região Sul (Tabela 4), enquanto que na Região Nordeste (Tabela 5) esteve associada à diminuição da escolaridade, pior autopercepção de saúde, não ter limitação para três ou mais atividades da vida diária e ao modelo PSF.

Prevalência de uso de serviços ambulatoriais nos últimos trinta dias entre idosos $(\mathrm{N}=3.994)$ * nas regiões Sul e Nordeste do Brasil. Projeto de Expansão e Consolidação do Saúde da Família (PROESF), 2005.

\begin{tabular}{|c|c|c|c|c|c|c|c|}
\hline \multirow[t]{2}{*}{ Variável } & \multicolumn{2}{|c|}{ Total } & \multicolumn{2}{|c|}{ Sul } & \multicolumn{2}{|c|}{ Nordeste } & \multirow{2}{*}{$\begin{array}{c}\text { Valor } \\
\text { de } p \text { ** }\end{array}$} \\
\hline & $\mathrm{n}$ & $\%$ & n & $\%$ & $\mathrm{n}$ & $\%$ & \\
\hline Uso de serviços ambulatoriais & & & & & & & $<0,001$ \\
\hline Total & 3.994 & 100,0 & 1.887 & 100,0 & 2.107 & 100,0 & \\
\hline Não usou & 2.701 & 67,6 & 1.214 & 64,3 & 1.487 & 70,6 & \\
\hline Unidade básica de saúde de abrangência & 643 & 16,1 & 332 & 17,6 & 311 & 14,8 & \\
\hline Outros locais & 650 & 16,3 & 341 & 18,1 & 309 & 14,6 & \\
\hline
\end{tabular}

* Houve perda de informações em nove indivíduos da amostra, quatro na Região Sul e cinco na Região Nordeste;

** Valor de $p$ do teste de qui-quadrado para heterogeneidade de proporções. 
Tabela 3

Análise bruta de consultas médicas ambulatoriais nos últimos trinta dias por idosos $(\mathrm{N}=3.994)$ * em municípios com mais de 100 mil habitantes no Sul e Nordeste do Brasil. Projeto de Expansão e Consolidação do Saúde da Família (PROESF), 2005.

\begin{tabular}{|c|c|c|c|c|c|c|c|c|}
\hline \multirow[t]{3}{*}{ Variáveis } & \multicolumn{4}{|c|}{ Região Sul ( $n=1.887$ ) } & \multicolumn{4}{|c|}{ Região Nordeste $(n=2.107)$} \\
\hline & \multirow[t]{2}{*}{$\begin{array}{c}\text { Não } \\
\text { consultou } \\
\text { n (\%) }\end{array}$} & \multirow[t]{2}{*}{$\begin{array}{l}\text { Consultou na } \\
\text { unidade básica } \\
\text { de saúde de } \\
\text { abrangência } \\
\text { n (\%) }\end{array}$} & \multirow[t]{2}{*}{$\begin{array}{l}\text { Consultou } \\
\text { em outro } \\
\text { local } \\
\text { n (\%) }\end{array}$} & \multirow[t]{2}{*}{$\begin{array}{c}\text { Valor } \\
\text { de } \\
p * \star\end{array}$} & \multirow[t]{2}{*}{$\begin{array}{c}\text { Não } \\
\text { consultou } \\
\text { n (\%) }\end{array}$} & \multirow[t]{2}{*}{$\begin{array}{l}\text { Consultou na } \\
\text { unidade básica } \\
\text { de saúde de } \\
\text { abrangência } \\
\text { n (\%) }\end{array}$} & \multirow[t]{2}{*}{$\begin{array}{l}\text { Consultou } \\
\text { em outro } \\
\text { local } \\
\text { n (\%) }\end{array}$} & \multirow[t]{2}{*}{$\begin{array}{c}\text { Valor } \\
\text { de } \\
p^{* \star}\end{array}$} \\
\hline & & & & & & & & \\
\hline Sexo & & & & 0,034 & & & & 0,997 \\
\hline Masculino & $483(67,9)$ & $109(15,3)$ & $119(16,7)$ & & $593(70,5)$ & $124(14,7)$ & $124(14,7)$ & \\
\hline Feminino & $731(62,2)$ & $223(19,0)$ & $222(18,9)$ & & $849(70,6)$ & $187(14,8)$ & $185(14,6)$ & \\
\hline Cor da pele & & & & 0,354 & & & & 0,042 \\
\hline Branca & $993(63,7)$ & $279(17,9)$ & $287(18,4)$ & & $851(69,4)$ & $173(14,1)$ & $202(16,5)$ & \\
\hline Parda & $110(65,9)$ & $33(20,0)$ & $24(14,4)$ & & $384(70,6)$ & $90(16,5)$ & $70(12,9)$ & \\
\hline Preta & $106(68,4)$ & $20(12,9)$ & $29(18,7)$ & & $248(74,9)$ & $47(14,2)$ & $36(10,9)$ & \\
\hline Situação conjugal & & & & 0,845 & & & & 0,300 \\
\hline Casado(a)/Com companheiro(a) & $515(64,7)$ & $136(17,1)$ & $145(18,2)$ & & $625(69,6)$ & $138(15,4)$ & $135(15,0)$ & \\
\hline Viúvo(a) & $557(64,5)$ & $155(18,0)$ & $151(17,5)$ & & $646(70,6)$ & $127(13,9)$ & $142(15,5)$ & \\
\hline Separado(a)/Divorciado(a)/Solteiro(a) & $133(62,7)$ & $35(16,1)$ & $44(20,8)$ & & $204(73,1)$ & $45(16,1)$ & $30(10,8)$ & \\
\hline Idade (anos) & & & & 0,060 & & & & 0,717 \\
\hline $65-69$ & $425(66,8)$ & $115(18,1)$ & $96(15,1)$ & & $466(70,2)$ & $106(16,0)$ & $92(13,9)$ & \\
\hline $70-74$ & $321(60,3)$ & $99(18,6)$ & $112(21,1)$ & & $367(69,9)$ & $76(14,5)$ & $82(15,6)$ & \\
\hline $75-79$ & $229(62,1)$ & $64(17,3)$ & $76(20,6)$ & & $316(70,5)$ & $70(15,6)$ & $62(13,8)$ & \\
\hline 80 ou mais & $239(68,3)$ & $54(15,4)$ & $57(16,3)$ & & $338(71,9)$ & $59(12,6)$ & $73(15,3)$ & \\
\hline Escolaridade (anos) & & & & 0,134 & & & & $<0,001$ \\
\hline 9 ou mais & $45(60,0)$ & $10(13,3)$ & $20(26,7)$ & & $56(68,3)$ & $6(7,3)$ & $20(24,4)$ & \\
\hline $5-8$ & $230(66,5)$ & $55(15,9)$ & $61(17,6)$ & & $149(67,7)$ & $24(10,9)$ & $47(21,4)$ & \\
\hline $1-4$ & $436(63,5)$ & $114(16,6)$ & $137(19,9)$ & & $342(71,1)$ & $65(13,5)$ & $74(15,4)$ & \\
\hline 0 & $490(64,9)$ & $145(19,2)$ & $120(15,9)$ & & $914(71,2)$ & $210(16,4)$ & $160(12,5)$ & \\
\hline Renda familiar (quintil) & & & & 0,002 & & & & 0,001 \\
\hline $5^{\circ}$ & $240(62,7)$ & $50(13,1)$ & $93(24,3)$ & & $199(65,7)$ & $33(10,9)$ & $71(23,4)$ & \\
\hline $4^{\circ}$ & $247(66,4)$ & $62(16,7)$ & $63(16,9)$ & & $237(70,1)$ & $55(16,3)$ & $46(13,6)$ & \\
\hline $3^{\circ}$ & $278(64,1)$ & $84(19,4)$ & $72(16,6)$ & & $357(70,8)$ & $84(16,7)$ & $63(12,5)$ & \\
\hline $2^{\circ}$ & $119(68,4)$ & $23(13,2)$ & $32(18,4)$ & & $539(72,6)$ & $108(14,6)$ & $95(12,8)$ & \\
\hline $1^{\circ}$ & $330(63,0)$ & $113(21,6)$ & $81(15,5)$ & & $155(70,5)$ & $31(14,1)$ & $34(15,5)$ & \\
\hline Tabagismo & & & & 0,038 & & & & 0,008 \\
\hline Nunca fumou & $584(63,8)$ & $163(17,8)$ & $169(18,5)$ & & $568(69,5)$ & $119(14,6)$ & $130(15,9)$ & \\
\hline Ex-fumante & $430(62,1)$ & $125(18,1)$ & $137(19,8)$ & & $663(68,8)$ & $152(15,8)$ & $149(15,5)$ & \\
\hline Fumante & $200(71,9)$ & $44(15,8)$ & $34(12,2)$ & & $256(78,8)$ & $39(12,0)$ & $30(9,2)$ & \\
\hline Autopercepção de saúde & & & & $<0,001$ & & & & 0,001 \\
\hline Regular/Ruim & $695(60,9)$ & $207(18,1)$ & $239(21,0)$ & & $962(68,6)$ & $208(14,8)$ & $233(16,6)$ & \\
\hline Excelente/Muito boa/Boa & $515(70,1)$ & $123(16,7)$ & $97(13,2)$ & & $499(75,2)$ & $95(14,3)$ & $70(10,5)$ & \\
\hline Limitação para três ou mais & & & & $<0,001$ & & & & 0,016 \\
\hline \multicolumn{9}{|l|}{ 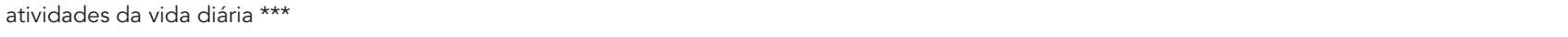 } \\
\hline Sim & $256(55,3)$ & $105(22,7)$ & $102(22,0)$ & & $409(68,7)$ & $79(13,3)$ & $107(18,0)$ & \\
\hline Não & $913(67,2)$ & $219(16,1)$ & $227(16,7)$ & & $1.035(71,2)$ & $226(15,5)$ & $193(13,3)$ & \\
\hline Tipo de unidade básica de saúde & & & & 0,694 & & & & $<0,001$ \\
\hline Tradicional & $506(64,7)$ & $131(16,8)$ & $145(18,5)$ & & $421(71,2)$ & $56(9,5)$ & $114(19,3)$ & \\
\hline Programa Saúde da Família & $708(64,1)$ & $201(18,2)$ & $196(17,7)$ & & $1.066(70,3)$ & $255(16,8)$ & $195(12,9)$ & \\
\hline
\end{tabular}

* Houve perda de informações em nove indivíduos da amostra, quatro na Região Sul e cinco na Região Nordeste;

** Valor de $\mathrm{p}$ do teste de qui-quadrado para heterogeneidade de proporções;

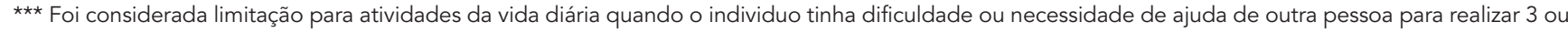
mais dentre 12 atividades - tomar banho, alimentar-se, vestir-se, usar o banheiro, caminhar, transferir-se da cama para uma cadeira, lavar o rosto, empurrar um objeto grande ( uma poltrona, por exemplo), carregar $5 \mathrm{~kg}$, agachar-se, levantar os braços acima da cabeça e lidar com objetos pequenos (uma chave, por exemplo). 
Fatores associados a consultas médicas em unidades básicas de saúde de abrangência por idosos da Região Sul do Brasil ( $N$ = 673). Projeto de Expansão e Consolidação do Saúde da Família (PROESF), 2005.

\begin{tabular}{|c|c|c|c|c|c|c|}
\hline \multirow[t]{2}{*}{ Nível * } & \multirow[t]{2}{*}{ Variável } & \multirow[b]{2}{*}{$\%$} & \multicolumn{2}{|c|}{ Análise bruta } & \multicolumn{2}{|c|}{ Análise ajustada } \\
\hline & & & RP (IC95\%) & Valor de $p$ & RP (IC95\%) & Valor de $p$ \\
\hline \multirow[t]{17}{*}{1} & Total & 49,3 & & & & \\
\hline & Idade (anos) & & & 0,234 ** & & $0,145 * \star$ \\
\hline & $65-69$ & 54,5 & 1,00 & & 1,00 & \\
\hline & $70-74$ & 46,9 & $0,95(0,89-1,02)$ & & $0,95(0,89-1,01)$ & \\
\hline & $75-79$ & 45,7 & $0,94(0,88-1,01)$ & & $0,93(0,87-1,00)$ & \\
\hline & 80 ou mais & 48,7 & $0,96(0,89-1,04)$ & & $0,96(0,88-1,03)$ & \\
\hline & Escolaridade (anos) & & & 0,031 ** & & $0,061 * \star$ \\
\hline & 9 ou mais & 33,3 & 1,00 & & 1,00 & \\
\hline & $5-8$ & 47,4 & $1,11(0,98-1,25)$ & & $1,09(0,97-1,23)$ & \\
\hline & $1-4$ & 45,4 & $1,09(0,97-1,22)$ & & $1,07(0,96-1,20)$ & \\
\hline & 0 & 54,7 & $1,16(1,03-1,31)$ & & $1,14(1,01-1,28)$ & \\
\hline & Renda familiar (quintil) & & & 0,001 ** & & 0,002 ** \\
\hline & $5^{\circ}$ & 35,0 & 1,00 & & 1,00 & \\
\hline & $4^{\circ}$ & 49,6 & $1,11(1,03-1,20)$ & & $1,10(1,02-1,20)$ & \\
\hline & $3^{\circ}$ & 53,9 & $1,14(1,06-1,23)$ & & $1,13(1,04-1,22)$ & \\
\hline & $2^{\circ}$ & 41,8 & $1,05(0,94-1,17)$ & & $1,02(0,91-1,14)$ & \\
\hline & $1^{\circ}$ & 58,3 & $1,17(1,09-1,26)$ & & $1,17(1,08-1,26)$ & \\
\hline \multirow[t]{3}{*}{3} & Autopercepção de saúde & & & 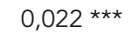 & & $0,004^{\star \star \star}$ \\
\hline & Regular/Ruim & 41,8 & 1,00 & & 1,00 & \\
\hline & Excelente/Muito boa/Boa & 58,3 & $1,06(1,01-1,12)$ & & $1,08(1,03-1,14)$ & \\
\hline \multirow[t]{3}{*}{4} & Tipo de unidade básica de saúde & & & $0,510 * \star \star$ & & 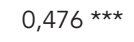 \\
\hline & Tradicional & 47,5 & 1,00 & & 1,00 & \\
\hline & Programa Saúde da Família & 50,6 & $1,02(0,96-1,09)$ & & $1,02(0,96-1,09)$ & \\
\hline
\end{tabular}

RP (IC95\%): razão de prevalência e intervalo de 95\% de confiança.

* O nível dois não foi apresentado porque a variável tabagismo não permaneceu no modelo final;

** Valor de $p$ do teste de Wald para tendência linear;

*** Valor de $\mathrm{p}$ do teste de Wald para heterogeneidade.

\section{Discussão}

A baixa densidade de indivíduos elegíveis por domicílio permitiu uma distribuição da amostra na área de abrangência das unidades básicas de saúde. A amostra estudada é representativa da população-alvo do estudo: idosos residentes em áreas de abrangência de unidades básicas de saúde de municípios com população a partir de 100 mil habitantes nas regiões Sul e Nordeste do Brasil. O pequeno percentual de indivíduos que responderam ao instrumento pelos idosos selecionados é um indicativo da confiabilidade das informações coletadas.

O critério de inclusão dos idosos a partir de 65 anos de idade, embora em conflito com a definição da OMS que recomenda o ponto de corte a partir de 60 anos para os países em desenvolvimento, proporciona comparabilidade com dados internacionais.
A comparação dos dados demográficos da amostra com os do Censo de 2000 pode auxiliar na caracterização dessa população. A amostra apresentou maior percentual de idosos do sexo feminino, viúvos(as) e de indivíduos a partir de 75 anos nas regiões Sul e Nordeste em relação aos dados do Censo Demográfico 2000 (Instituto Brasileiro de Geografia e Estatística; http:// www.ibge.gov.br) para a mesma idade. A pior escolaridade no Nordeste em relação ao Sul havia sido verificada no censo de 2000 , porém a proporção de ausência de escolaridade em ambas as regiões foi ainda maior do que a encontrada naquele inquérito.

Um estudo de base domiciliar realizado na Região Nordeste também encontrou maior percentual de idosos do sexo feminino e na faixa etária com oitenta anos ou mais do que os dados do último Censo Demográfico 13. 
Fatores associados a consultas médicas em unidades básicas de saúde de abrangência por idosos da Região Nordeste do Brasil ( $\mathrm{N}=620$ ). Projeto de Expansão e Consolidação do Saúde da Família (PROESF), 2005.

\begin{tabular}{|c|c|c|c|c|c|c|}
\hline \multirow[t]{2}{*}{ Nível * } & \multirow[t]{2}{*}{ Variável } & \multirow[b]{2}{*}{$\%$} & \multicolumn{2}{|c|}{ Análise bruta } & \multicolumn{2}{|c|}{ Análise ajustada } \\
\hline & & & RP (IC95\%) & Valor de $\mathrm{p}$ & RP (IC95\%) & Valor de $p$ \\
\hline \multirow[t]{15}{*}{1} & Total & 50,2 & & & & \\
\hline & Situação conjugal & & & 0,143 ** & & $0,125 * \star$ \\
\hline & Casado(a)/Com companheiro(a) & 50,6 & 1,00 & & 1,00 & \\
\hline & Viúvo(a) & 47,2 & $0,98(0,92-1,04)$ & & $1,00(0,94-1,06)$ & \\
\hline & Separado(a)/Divorciado(a)/Solteiro(a) & 60,0 & $1,06(0,99-1,14)$ & & $1,07(1,00-1,15)$ & \\
\hline & Idade (anos) & & & 0,228 *** & & $0,100 * \star \star$ \\
\hline & $65-69$ & 53,5 & 1,00 & & 1,00 & \\
\hline & $70-74$ & 48,1 & $0,96(0,90-1,04)$ & & $0,96(0,90-1,03)$ & \\
\hline & $75-79$ & 53,0 & $1,00(0,93-1,07)$ & & $0,97(0,90-1,04)$ & \\
\hline & 80 ou mais & 44,7 & $0,94(0,87-1,02)$ & & $0,93(0,86-1,01)$ & \\
\hline & Escolaridade (anos) & & & $<0,001$ *** & & $<0,001 * * *$ \\
\hline & 9 ou mais & 23,1 & 1,00 & & 1,00 & \\
\hline & $5-8$ & 33,2 & $1,09(0,93-1,27)$ & & $1,13(0,96-1,33)$ & \\
\hline & $1-4$ & 46,8 & $1,19(1,04-1,37)$ & & $1,23(1,06-1,43)$ & \\
\hline & 0 & 56,8 & $1,27(1,13-1,44)$ & & $1,33(1,17-1,52)$ & \\
\hline \multirow[t]{7}{*}{3} & Autopercepção de saúde & & & 0,009 ** & & 0,021 \\
\hline & Regular/Ruim & 47,2 & 1,00 & & 1,00 & \\
\hline & Excelente/Muito boa/Boa & 57,6 & $1,07(1,02-1,13)$ & & $1,06(1,01-1,11)$ & \\
\hline & Limitação para três ou mais & & & & & \\
\hline & atividades da vida diária & & & 0,007 ** & & 0,004 ** \\
\hline & Sim & 42,5 & 1,00 & & 1,00 & \\
\hline & Não & 53,9 & $1,08(1,02-1,14)$ & & $1,09(1,03-1,16)$ & \\
\hline \multirow[t]{3}{*}{4} & Tipo de unidade básica de saúde & & & 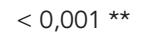 & & $<0,001$ ** \\
\hline & Tradicional & 32,9 & 1,00 & & 1,00 & \\
\hline & Programa Saúde da Família & 56,7 & $1,18(1,11-1,26)$ & & $1,18(1,11-1,26)$ & \\
\hline
\end{tabular}

RP (IC95\%): razão de prevalência e intervalo de 95\% de confiança.

* O nível dois não foi apresentado porque a variável tabagismo não permaneceu no modelo final;

** Valor de $\mathrm{p}$ do teste de Wald para heterogeneidade;

$\star \star \star$ Valor de $\mathrm{p}$ do teste de Wald para tendência linear.

A maior prevalência dessas características na amostra pode levar a um aumento do uso dos serviços de saúde porque estudos anteriores identificaram associação dessas categorias com uma maior utilização dos serviços de saúde 14,15.

A maior concentração de indivíduos idosos nos quintis de menor renda no Nordeste em relação ao Sul é compatível com dados da Pesquisa Nacional por Amostra de Domicílios de 2004 (PNAD 2004) 16.

O percentual de indivíduos que nunca fumaram foi menor na Região Nordeste. A menor escolaridade dos idosos nessa região pode ser uma explicação provável para essa ocorrência já que a associação negativa entre escolaridade e tabagismo foi observada em estudo de base populacional de idosos no Brasil 17.
Metodologias diversas dificultam a comparação dos indicadores de necessidades em saúde da amostra com outros estudos. Embora a PNAD 2003 tenha utilizado uma escala diferente para examinar a autopercepção de saúde, verifica-se que ao se agrupar as categorias regular/ruim/ muito ruim da população idosa naquele estudo se obtém percentual semelhante ao encontrado neste estudo para a categoria regular/ruim 18 . A prevalência de dificuldade para caminhar $100 \mathrm{~m}$, pesquisada na PNAD 2003, foi similar à encontrada neste estudo para a limitação para 3 ou mais de 12 atividades da vida diária 19,20.

O modelo PSF foi mais freqüente na Região Nordeste do que na Região Sul o que é condizente com os dados do Ministério da Saúde que justificam a maior implantação desta estratégia 
naquela região, devido à existência de piores taxas de morbidade e mortalidade populacional e maior carência de serviços de saúde em geral 21 .

\section{Uso de serviços ambulatoriais de saúde}

Os resultados deste estudo demonstraram que o uso dos serviços ambulatoriais foi maior na Região Sul do que na Região Nordeste, apesar da maior proporção de idosos com indicadores de necessidades de cuidados de saúde nesta região. Essa desigualdade no uso dos serviços de saúde entre as duas regiões geográficas pode se dever à maior dificuldade de acesso e/ou menor oferta de serviços na Região Nordeste. A menor escolaridade e renda familiar dos idosos do Nordeste podem dificultar o acesso aos serviços de saúde pela falta de informação dos serviços disponíveis e/ ou pelo custo do deslocamento até eles 22 . Quanto à oferta de serviços, uma avaliação da distribuição da rede urbana de serviços públicos de saúde em todos os níveis com base em informações municipais identificou na Região Sul uma boa distribuição espacial dos serviços de saúde, enquanto na Região Nordeste há uma concentração de recursos humanos e de infraestrutura dos serviços de saúde apenas nas capitais dos estados 23 .

A utilização de serviços ambulatoriais por idosos mostrou estar associada tanto na Região Sul quanto na Região Nordeste a ser ex-fumante. Estudos anteriores têm demonstrado que aqueles que abandonaram o tabagismo e aqueles que nunca fumaram utilizam mais os serviços de saúde, o que tem sido atribuído ao maior cuidado destes indivíduos com a saúde em relação aos tabagistas 24,25. A associação com ex-fumantes pode ter um viés de causalidade reversa na medida em que o abandono do tabagismo pode ser decorrência de recomendação médica. Por outro lado, a falta de associação entre os fumantes atuais e o uso de serviços de saúde pode se dever ao viés de sobrevivência, uma vez que a mortalidade entre os fumantes é maior do que nos outros grupos 26 .

Os idosos com autopercepção de saúde regular/ruim usaram mais os serviços ambulatoriais tanto no Sul quanto no Nordeste. A autopercepção de saúde do indivíduo é considerada um bom indicador de necessidade de cuidados de saúde 27 , e o mesmo resultado foi observado em revisões sistemáticas de estudos com populações de adultos 14 e de idosos 27,28.

Os idosos com dificuldade para realizar três ou mais atividades da vida diária utilizaram mais os serviços ambulatoriais de saúde apenas na Região Sul. A associação de limitações funcionais com maior uso de consultas médicas entre os idosos tem sido encontrada em outros estudos 29, 30, 31, 32. A ausência dessa associação no Nordeste indica a maior dificuldade de acesso dos idosos portadores dessas condições de saúde aos serviços e/ou o despreparo dos serviços de saúde para o manejo adequado dessas morbidades 22 .

A análise ajustada do modelo para uso de serviços ambulatoriais por idosos mostrou que nas duas regiões estudadas ele se associou apenas a variáveis indicadoras de necessidade de cuidados em saúde, demonstrando adequação dos atendimentos realizados conforme modelo proposto por Aday \& Andersen 33 .

\section{Consulta em Atenção Básica à Saúde}

Utilizando o mesmo modelo de análise para avaliar os determinantes da escolha de consulta em unidade básica de saúde de abrangência foi observado que, no Sul, ele associou-se à menor renda familiar e melhor autopercepção de saúde, e no Nordeste, à menor escolaridade, maior autonomia para atividades da vida diária, melhor autopercepção de saúde e ser vinculado à unidade básica de saúde com PSF.

A associação de consulta em unidade básica de saúde de abrangência com menor renda familiar no Sul e com menor escolaridade no Nordeste indica que os idosos com pior condição sócio-econômica estão tendo acesso aos serviços da atenção básica à saúde, o que contribui para a eqüidade em saúde. Resultado similar foi encontrado em estudo populacional, que comparou o uso de serviços de saúde por idosos nas redes pública e privada, o qual verificou que os idosos com maior renda familiar per capita utilizavam mais a rede suplementar de saúde 11 .

A evidência de que idosos portadores de maiores necessidades de cuidados de saúde procuram outros locais de atendimento em relação à unidade básica de saúde de abrangência sugere que a atenção básica à saúde não está preparada para prestar atendimento a estas condições de saúde. Dados do ELB do PROESF demonstraram que a infra-estrutura das unidades básicas de saúde e a capacitação dos profissionais de saúde, de ambos os modelos de atenção básica, são bastante precárias ${ }^{12}$. Para aumentar a captação desses indivíduos a rede básica necessita melhorar sua infra-estrutura, ter profissionais capacitados para lidar com as necessidades de saúde dos idosos e contar com o apoio de equipe multiprofissional (fisioterapeuta, nutricionista, fonoaudiólogo, ortopedista, neurologista, entre outros) quando o atendimento especializado é necessário 34. 
A associação de uso de unidade básica de saúde de abrangência por idosos com o modelo PSF apenas no Nordeste é explicada pela implantação e maior expansão do Programa nesta região devido à política de priorizar este modelo para as populações mais pobres ${ }^{21}$.

\section{Conclusões}

A discussão sobre a importância de melhorar o atendimento prestado aos idosos na atenção básica tem sido objeto de interesse da OMS e dos sistemas de saúde em todo o mundo. Cada país deverá encontrar a solução mais adequada ao seu contexto.

Este estudo mostrou que os idosos residentes em áreas de abrangência de unidades básicas de saúde da Região Nordeste, apesar de terem pior escolaridade e maior prevalência de indicadores de cuidados de saúde, têm uma utilização inferior e mais inadequada dos serviços ambulatoriais de saúde do que aqueles da Região Sul. Esses dados indicam provável carência na oferta dos serviços e dificuldade de acesso dos idosos aos serviços, principalmente os portadores de limitações funcionais. O uso da unidade básica de saúde de abrangência foi determinado por piores condições sócio-econômicas e melhores condições de saúde dos idosos, demonstrando que em ambas as regiões a atenção básica à saúde, embora promova a eqüidade, não está preparada para atender às necessidades de saúde dos idosos.

O sistema público de saúde deve aumentar a oferta de serviços e melhorar o acesso aos idosos, especialmente os portadores de limitações funcionais, na Região Nordeste. A saúde do idoso deve ser prioritária na atenção básica à saúde, que deve implementar ações para forne- cer atendimento adequado às necessidades de saúde deste grupo populacional.

\section{Limitações}

Este estudo tem um desenho transversal o que não permite averiguar relações de causa e efeito. No entanto, o objetivo deste trabalho foi identificar características relacionadas ao uso de serviços ambulatoriais e de atenção básica à saúde por idosos, o que é permitido pelo desenho do estudo. Embora o viés de causalidade reversa não possa ser descartado por ser um risco inerente ao desenho do estudo, o uso de recordatório em algumas questões tentou minimizá-lo.

A amostra do trabalho não foi de base populacional, porém o objetivo foi avaliar os idosos residentes em zonas urbanas periféricas, parcela mais vulnerável deste grupo populacional, o que justifica o processo de amostragem.

\section{Vantagens}

A amostra é representativa da população-alvo o que garantiu a validade interna deste estudo. Os resultados que foram semelhantes em ambas as regiões geográficas podem ser extrapolados para populações com as mesmas características daquelas do estudo.

A realização do trabalho em duas regiões geográficas diferentes permitiu a avaliação do desfecho em realidades distintas.

Os resultados do estudo apontam para um problema relevante na saúde pública: a atenção ao idoso na rede básica de saúde, o que deve contribuir para o planejamento de modificações que contemplem as necessidades de saúde desta população. 


\section{Resumo}

Este estudo avaliou a utilização de serviços ambulatoriais por idosos. Foram utilizados dados de 4.003 idosos do estudo de linha de base que avaliou o Projeto de Expansão e Consolidação do Saúde da Família (PROESF) em 41 municípios das regiões Sul e Nordeste do Brasil. O uso de serviços ambulatoriais foi maior e mais adequado às necessidades de saúde dos idosos no Sul do que no Nordeste. A atenção básica à saúde, em ambas as regiões, atendeu a demanda dos idosos em piores condições sócio-econômicas, porém aqueles com maiores necessidades de cuidados foram atendidos em outros locais. Os resultados indicam que é preciso aumentar a oferta de serviços ambulatoriais de saúde e garantir o acesso aos idosos, especialmente os portadores de limitações funcionais, na Região Nordeste. Em ambas as regiões a atenção básica à saúde, além de promover a eqüidade, deve ter abordagens direcionadas às necessidades de saúde dos idosos.

Serviços Ambulatoriais de Saúde; Avaliação de Programas e Projetos de Saúde; Atenção Primária à Saúde; Saúde do Idoso

\section{Colaboradores}

M. A. P. Rodrigues realizou a revisão de literatura, participou da elaboração dos instrumentos, processamento, análise e interpretação dos dados e redação final do artigo. L. A. Facchini participou na concepção, elaboração dos instrumentos, interpretação dos dados e revisão final do artigo. E. Tomasi, E. Thumé, D. S. Silveira e R. X. Piccini participaram da concepção, elaboração dos instrumentos e processamento dos dados. F. V. Siqueira coordenou o trabalho de campo do estudo e participou da elaboração dos instrumentos e processamento dos dados. V. M. V. Paniz participou da elaboração dos instrumentos, processamento dos dados e realizou o controle de qualidade da pesquisa. Todos os autores participaram da redação do artigo.

\section{Agradecimentos}

Este trabalho integra o componente 3 do Projeto de Expansão e Consolidação do Saúde da Família (PROESF), coordenado e financiado pelo Ministério da Saúde com recursos do Banco Mundial.

\section{Referências}

1. World Health Organization. Social development ageing: crisis or opportunity? Geneva: World Health Organization; 2000.

2. World Health Organization. Active ageing: towards age-friendly primary health care. Geneva: World Health Organization; 2004.

3. Brasil. Lei $\mathrm{n}^{\circ}$. 8.080, de 19 de setembro de 1990. Dispõe sobre as condições para a promoção, proteção e recuperação da saúde, a organização e o funcionamento dos serviços correspondentes. Diário Oficial da União 1990; 20 set.

4. Ministério da Saúde. Manual de organização da atenção básica. Brasília: Ministério da Saúde; 1999.

5. Agência Nacional de Saúde Suplementar. Lei no 9.656, de 3 de junho de 1998. http://www.ans. gov.br/portal/site/legislacao/legislacao_integra. asp?id_original=455 (acessado em 04/Ago/2007).

6. Ministério da Saúde. Política nacional de saúde do idoso. Diário Oficial da União 1999; 13 dez.
7. Ministério da Previdência Social. Lei no ${ }^{\circ} 10.741$ - estatuto do idoso. Diário Oficial da União 2003; 1 out.

8. Ministério da Saúde. Pacto pela saúde 2006. Diário Oficial da União 2006; 23 fev.

9. Lima-Costa MF, Matos DL, Camarano AA. Evolução das desigualdades sociais em saúde entre idosos e adultos brasileiros: um estudo baseado na Pesquisa Nacional por Amostra de Domicílios (PNAD 1998, 2003). Ciênc Saúde Coletiva 2006; 11:941-50.

10. Pinheiro RS, Travassos C. Estudo da desigualdade na utilização de serviços de saúde por idosos em três regiões da cidade do Rio de Janeiro. Cad Saúde Pública 1999; 15:487-96.

11. Bós AMG, Bós AJG. Determinantes na escolha entre atendimento de saúde privada e pública por idosos. Rev Saúde Pública 2004; 38:113-20. 
12. Facchini LA, Piccini RX, Tomasi E, Thumé E, Silveira DS, Siqueira FV, et al. Desempenho do PSF no Sul e no Nordeste do Brasil: avaliação institucional e epidemiológica da atenção básica à saúde. Ciênc Saúde Coletiva 2006; 11:669-81.

13. Coelho Filho JM, Ramos LR. Epidemiologia do envelhecimento no Nordeste do Brasil: resultados de inquérito domiciliar. Rev Saúde Pública 1999; 33:445-53.

14. Mendoza-Sassi R, Béria JU. Utilización de los servicios de salud: una revisión sistemática sobre los factores relacionados. Cad Saúde Pública 2001; 17:819-32.

15. Sawyer DO, Leite IC, Alexandrino R. Perfis de utilização de serviços de saúde no Brasil. Ciênc Saúde Coletiva 2002; 7:757-76.

16. Instituto Brasileiro de Geografia e Estatística. Pesquisa Nacional por Amostra de Domicílios, 2004. Rio de Janeiro: Instituto Brasileiro de Geografia e Estatística; 2005.

17. Peixoto SV, Firmo JOA, Lima-Costa MF. Factors associated to smoking habit among older adults (The Bambuí Health and Aging Study). Rev Saúde Pública 2005; 39:746-53.

18. Dachs JNW, Santos APR. Auto-avaliação do estado de saúde no Brasil: dados da PNAD/2003. Ciênc Saúde Coletiva 2006; 11:887-94.

19. Parahyba MI, Simões CCS. A prevalência de incapacidade funcional em idosos no Brasil. Ciênc Saúde Coletiva 2006; 11:967-74.

20. Ribeiro MCSA, Barata RB, Almeida MF, Silva ZP. Perfil sociodemográfico e padrão de utilização de serviços de saúde para usuários e não-usuários do SUS - PNAD 2003. Ciênc Saúde Coletiva 2006; 11:1011-22.

21. Departamento de Atenção Básica, Secretaria de Políticas de Saúde. Programa Saúde da Família. Rev Saúde Pública 2000; 34:316-9.

22. Taylor Jr. DH, Hoenig H. Access to health care services for the disabled elderly. Health Serv Res 2006; 41(3 Pt 1):743-58.

23. Guimarães C, Amaral P, Simões R. Rede urbana da oferta de serviços de saúde: uma análise multivariada macro regional - Brasil, 2002. In: XV Encontro Nacional de Estudos Populacionais. http://www. abep.nepo.unicamp.br/encontro2006/docspdf/ ABEP2006_422.pdf (acessado em 04/Ago/2007).
24. Adab P, McGhee SM, Hedley AJ, Lam TH. Smoking, respiratory disease and health service utilisation: the paradox. Soc Sci Med 2005; 60:483-90.

25. Capilheira MF, Santos IS. Fatores individuais associados à utilização de consultas médicas por adultos. Rev Saúde Pública 2006; 40:436-43.

26. Ogren M, Hedblad B, Janzon L. Biased risk factor assessment in prospective studies of peripheral arterial disease due to change in exposure and selective mortality of high-risk individuals. J Cardiovasc Risk 1996; 3:523-8.

27. Lima-Costa MF, Firmo JOA, Uchôa E. A estrutura de auto-avaliação de saúde entre idosos: Projeto Bambuí. Rev Saúde Pública 2004; 38:827-34.

28. Borrás JM. La utilització dels serveis sanitaris. Gac Sanit 1994; 8:30-49.

29. Al Snih S, Markides KS, Ray LA, Freeman JL, Ostir GV, Goodwin JS. Predictors of healthcare utilization among older Mexican Americans. Ethn Dis 2006; 16:640-6.

30. Chou KL, Chi I. Factors associated with the use of publicly funded services by Hong Kong Chinese older adults. Soc Sci Med 2004; 58:1025-35.

31. Otero A, Zunzunegui MV, Rodriguez-Laso A, Aguilar MD, Lazaro P. Volumen y tendencias de la dependencia asociada al envejecimiento en la población española. Rev Esp Salud Pública 2004; 78:201-13.

32. Walter-Ginzburg A, Chetrit A, Medina C, Blumstein T, Gindin J, Modan B. Physician visits, emergency room utilization, and overnight hospitalization in the old-old in Israel: the cross-sectional and longitudinal aging study (CALAS). J Am Geriatr Soc 2001; 49:549-56

33. Aday LA, Andersen RM. Equity of access to medical care: a conceptual and empirical overview. Med Care 1981; 19(12 Suppl):4-27.

34. Lollar DJ, Crews JE. Redefining the role of public health in disability. Annu Rev Public Health 2003; 24:195-208.

Recebido em 23/Out/2007

Versão final reapresentada em 10/Mar/2008

Aprovado em 28/Mar/2008 